\title{
Controlling of a ROS-based robotic system in accordance to the assist-as-needed principle in end-effector based rehabilitation systems
}

Sebastian Becker, Department of Rehabilitation and Prevention Engineering, Institute of Applied Medical Engineering, RWTH Aachen University, Aachen, Germany, becker@ame.rwth-aachen.de

Wiebke Hinterlang, Department of Rehabilitation and Prevention Engineering, Institute of Applied Medical Engineering, RWTH Aachen University, Aachen, Germany, wiebke.hinterlang@rwth-aachen.de

Tim Eschert, Department of Rehabilitation and Prevention Engineering, Institute of Applied Medical Engineering, RWTH Aachen University, Aachen, Germany, tim.eschert@rwth-aachen.de

Catherine Disselhorst-Klug, Department of Rehabilitation and Prevention Engineering, Institute of Applied Medical Engineering, RWTH Aachen University, Aachen, Germany, disselhorst-klug@ame.rwth-aachen.de

Stroke is one of the most frequent diseases among the elderly and often leads to an ongoing failure of functions in the central nervous system. Due to the plasticity of the brain affected may regain lost motor function by repetitive training. Robotic devices can be an approach to accelerate the rehabilitation process by maximizing patients' training intensity. End-effector based robotic systems are particularly suitable for this purpose and often an advantage over exoskeletons since the proximal part of the upper limb remains under the control of the patient. Furthermore, the integration of the assist-as-needed principle (AAN) into these devices enables individualized, adaptable robotic support to patients during therapy. In this study an end-effector based robotic rehabilitation device based on the Robot Operating System (ROS) framework is introduced. The system allows patients to perform 3-dimensional movements without a therapist's assistance. With regard to the AAN, focus was based on impedance control and an additional real-time adaption of the impedance control parameters by using a feedback loop. 10 healthy subjects took part in this study to evaluate the overall concept with regard to usability and quality of the supported movement. Hence, the three most promising adaption models of AAN (without adaption, adaption according to position and time, adaption according to velocity) under three different levels of movement support $(0 \%, 50 \%, 100 \%)$ were investigated by administering a self-designed questionnaire and the robot kinematics. The results showed no significant differences between the three different adaption models of AAN. However, the subjective assessment of the movements was in keeping with robot kinematics and the control approaches as well as the overall system have experienced remarkable support. 


\section{Biomechanics of a novel extra articular implant for younger patients with knee osteoarthritis}

Mehdi Saeidi, Department of Mechanical Engineering, Auckland University of Technology, Auckland, New Zealand, msaeidi@aut.ac.nz

Maziar Ramezani, Department of Mechanical Engineering, Auckland University of Technology, Auckland, New Zealand, maziar.ramezani@aut.ac.nz

Piaras Kelly, Department of Engineering Science, University of Auckland, Auckland, New Zealand, pa.kelly@auckland.ac.nz

Thomas Neitzert, Department of Mechanical Engineering, Auckland University of Technology, Auckland, New Zealand, thomas.neitzert@aut.ac.nz

Most patients with moderate to end stage Knee Osteoarthritis (OA) undergo Total Knee Arthroplasty (TKA) eventually, and the main reason for use of other methods, specifically in younger patients, is to postpone this last resort. TKA is typically recommended for people over 65 years old; however, research shows that more than $40 \%$ of TKAs have been performed before this age. Therefore, this study aimed to develop a new implant in order to partially unload the knee joint, targeted at early-to-moderate grades of OA and which would be especially appropriate for younger patients. The implant was designed on the surfaces of MRI-scanned bones via SolidWorks ${ }^{\circledR}$, thus it can be considered to be patientspecific. A prototype of the implant was made and attached to a cadaveric knee joint. To fix the implant to the bone, porous screws as well as an osteointegrated lattice structure over the back of the implant were considered. A static Finite Element Analysis (FEA) was conducted in Abaqus ${ }^{\circledR}$ for the extended knee position, before and after attaching the implant, in order to evaluate the stress distribution on the tibiofemoral interfaces. Material properties of the hard and soft tissues were accurately modeled based on the literature. Surgery of the proposed implant does not require sacrificing soft and hard tissues of the knee joint, i.e. ligaments or bones. The von Mises stresses and contact pressure were reduced in the medial compartment by $40 \%$ and $35 \%$, respectively, after implementing the implant. Results of the study show that the proposed implant removes excessive load from the knee and so slows progression of OA, creating a suitable environment in which osteoarthritic lesions might repair. Apart from the beneficial fact that surgery of the implant is reversible, it might be applied as an adjuvant treatment with other therapies as well. 


\section{Effects of the medial and lateral tibial slope on knee joint kinematics in total knee arthroplasty}

Malte Asseln, Chair of Medical Engineering, Helmholtz-Institute for Biomedical Engineering, RWTH Aachen University, Aachen, Germany, e-Mail: asseln@hia.rwth-aachen.de

Mark Verjans, Chair of Medical Engineering, Helmholtz-Institute for Biomedical Engineering, RWTH Aachen University, Aachen, Germany, e-Mail: verjans@hia.rwth-aachen.de

Luisa Berger, Chair of Medical Engineering, Helmholtz-Institute for Biomedical Engineering, RWTH Aachen University, Aachen, Germany, e-Mail: luisa.berger@rwth-aachen.de

Klaus Radermacher, Chair of Medical Engineering, Helmholtz-Institute for Biomedical Engineering, RWTH Aachen University, Aachen, Germany, e-Mail: radermacher@hia.rwth-aachen.de

Implant design in total knee arthroplasty is a key factor for the recovery of function and mobility of the joint. However, there is a lack of knowledge on the complex relationship between design features and the biomechanical situation. The posterior inclination of the tibial plateau (tibial slope) has a known functional impact on knee joint kinematics. In the native knee it shows high inter-individual differences, which is inadequately considered in total knee arthroplasty. We investigated the effects of the separate and combined variation of the medial and lateral tibial slope on knee joint kinematics in an in vitro setup. Therefore, the medial and lateral inserts of a knee implant were parameterised and the initial slope was gradually varied to $+4^{\circ},+2^{\circ}$ (posterior) and $-2^{\circ},-4^{\circ}$ (anterior). The variations were manufactured by using polymer based rapid prototyping. The effects on semi-active tibiofemoral and patellofemoral kinematics were studied and quantified in terms of the Root Mean Square Error and Pearson's Correlation coefficient. The overall largest effect was observed on tibiofemoral internal-external rotation by the variation of the lateral insert. The anterior-posterior translation was predominantly effected by the combined variation. Nevertheless, the lateral variation showed greater influence than medial. In contrast, changes in patellofemoral kinematics were small. Our findings suggest that in total knee arthroplasty the lateral tibial slope has a larger functional effect than the medial tibial slope. Thus, functional design optimization should focus on the lateral insert. 


\section{In vivo preclinical application of an active external fixator to investigate the in- fluence of local mechanical conditions on the fracture healing}

Jan Barcik, AO Research Institute Davos, Davos, Switzerland, jan.barcik@aofoundation.org

Manuela Ernst, AO Research Institute Davos, Davos, Switzerland, manuela.ernst@aofoundation.org

Linda Freitag, AO Research Institute Davos, Davos, Switzerland, linda.freitag@aofoundation.org

Constantin E. Dlaska, Charité CMSC Berlin, Berlin, Germany, constantin.dlaska@charite.de

Boyko Gueorguiev-Rüegg, AO Research Institute Davos, Davos, Switzerland, boyko.gueorguiev@aofoundation.org

Devakar Epari, Queensland University of Technology, Brisbane, Australia, d.epari@qut.edu.au

Markus Windolf, AO Research Institute Davos, Davos, Switzerland, markus.windolf@aofoundation.org

Bone fracture healing is influenced by local mechanical conditions present in the fracture gap. Despite decades of research on bone healing, optimal mechanical conditions promoting bone healing remain unknown. In this project we are aiming at developing a system which installs well-controlled mechanical conditions in the fracture gap and evaluates the resultant healing progression. A deeper understanding of the influence of the mechanical environment on fracture healing shall allow to optimize implant design and rehabilitation protocols to decrease the number of patients with delayed bone union or non-unions.

We applied an active external fixator capable of establishing specific mechanical conditions in a tibial osteotomy in sheep. A linear actuator embedded in the fixator compresses the fracture gap to mimic an effect of weight bearing independently from the animal's body weight. We developed a custom-made controller that allows to program the amplitude of fracture stimulation, stimulation strain rate, and the temporal distribution of the stimulation cycles. For all load cycles, axial force and displacement within the fracture gap is recorded to monitor stiffness changes of the repair tissue. The fixation system was implanted in two sheep mimicking different rehabilitation protocols i.e. immediate and delayed weight bearing.

The active fixator and controller kept functioning over the whole course of the experiment. Both animals tolerated the fixation system well. A clear difference in the healing onset was observed between both animals.

In this project we developed and tested a research implant system that collects experimental data describing the influence of mechanical conditions on fracture healing in vivo. We present a very flexible tool that allows to apply vast range of different loading scenarios to investigate and characterize the optimal mechanical conditions to promote fracture healing. 


\section{Interaction of synergistic and antagonistic muscles of elbow joint during activi- ties of daily living in healthy children}

Alberto-Isaac Perez-Sanpablo, Human Motion Analysis Lab, Mexican National Institute of Rehabilitation/Automatic Control Department, CINVESTAV, Mexico City, Mexico, albperez@inr.gob.mx; alberto.perez@cinvestav.mx

Elisa Romero-Avila, Rehabilitation and Prevention Engineering, RWTH-Aachen University, Aachen, Germany, romero@ame.rwth-aachen.de

Alicia Meneses-Peñaloza, Pediatrics Rehabilitation Department, Mexican National Institute of Rehabilitation, Mexico City, Mexico, ameneses@inr.gob.mx

Maria-Elena Arellano-Saldaña, Pediatrics Rehabilitation Department, Mexican National Institute of Rehabilitation, Mexico City, Mexico, marellano@inr.gob.mx

Catherine Disselhorst-Klug, Rehabilitation and Prevention Engineering, RWTH-Aachen University, Aachen, Germany, disselhorst-klug@ame.rwth-aachen.de

Josefina Gutierrez-Martinez, Medical Engineering Department, Mexican National Institute of Rehabilitation, Mexico City, Mexico,josefina_gutierrez@hotmail.com

Juan-Manuel Ibarra-Zannatha, Automatic Control Department, CINVESTAV, Mexico City, Mexico, jibarra@cinvesvtav.mx

The aim of the study is to analyze the interaction of biceps brachii, brachioradialis, triceps medialis and triceps lateralis during activities of daily living (ADL) tasks performed by typically developed children. Surface electromyography (sEMG) of 8 healthy children (mean age 10 years old, 5 male, 4 female) during dynamic movements with different combinations of range of motion, angular velocities and loads were registered on previously mentioned muscles after signature of informed consent and assent. Elbow kinematics was measured by using an electrogoniometer. Measuring protocol was approved by institutional ethics and research comittee. The measurements were conducted during ADL corresponding to maneuvers of a previously proposed functional assessment battery for elbow functionality for children. Maneuvers include lifting an object to waist level, placing an object with both hands above the head, throwing an object, using a knife to cut food, grasping and drinking from a glass and hammering. Elbow angle was low-pass filtered. Elbow velocity was calculates as the low-pass filtered version of elbow position gradient. Maximum elbow velocity was $377 \%$ and $748 \%$ s for elbow flexion and extension respectively. Electromiographic signals were bandpass filtered, rectified, smoothed and normalized to obtain muscle activation profiles. Muscular activation of each muscle during concentric and eccentric contractions were divided into five joint angle categories $\left(0^{\circ}-130^{\circ}\right)$ and five joint angular velocity categories $(0 \% \mathrm{~s}-800 \% \mathrm{~s})$ based on elbow kinematic data. Significant differeces of muscular activation were observed for activity, muscle, angle and velocity in both concentric and eccentric contractions $(\mathrm{p}<0.05)$. All muscles presented higher activation levels and synergistic muscular activation during concentric contraccions. Flexor muscles presented also synergistic activation during eccentric contractions. However triceps medialis and triceps lateralis presented a different behavior at high joint angles during eccentric contraccions which suggest the use of different control strategies for extensor muscles during ADL. Research support CONACyT-BMBF-FONCICYT-267734. 


\section{Optimization of the cardiovascular stent design towards improved ex- pansion behaviour and radial stiffness properties}

Lisa Wiesent, Numerical Material Science Lab, Medical Device Lab, Regensburg Center of Biomedical Engineering, Ostbayerische Technische Hochschule Regensburg, lisa.wiesent@ oth-regensburg.de

Constantin Hupke, Medical Device Lab, Faculty of Mechanical Engineering, Ostbayerische Technische Hochschule Regensburg, constantin.hupke@st.oth-regensburg.de

Christian Balk, Medical Device Lab, Faculty of Mechanical Engineering, Ostbayerische Technische Hochschule Regensburg, christian.balk@st.oth-regensburg.de

Ulrich Schultheiß, Laboratory for Material Surface Layer Analytics, Faculty of Mechanical Engineering, Ostbayerische Technische Hochschule Regensburg, ulrich.schultheiss@oth-regensburg.de

Thomas Schratzenstaller, Medical Device Lab, Faculty of Mechanical Engineering, Regensburg Center of Biomedical Engineering, Ostbayerische Technische Hochschule Regensburg, thomas.schratzenstaller@oth-regensburg.de

Aida Nonn, Numerical Material Science Lab, Faculty of Mechanical Engineering, Ostbayerische Technische Hochschule Regensburg, aida.nonn@oth-regensburg.de

Coronary heart disease is one of the common cause of death in the industry countries. It is mainly treated by the implantation of a coronary stent. A major issue of stent treatment is in-stent restenosis, the re-narrowing of the vessel at the site of the implanted stent. In-stent restenosis is mainly related to vessel injuries during the expansion of the stent. Commonly stent expansion is initiated at the stent ends before advancing to the stent centre resulting in the usual dogbone shaped expansion behaviour. This leads to local stress concentrations and micro injuries of the vessel wall resulting in the proliferation of the vessel wall cells. To overcome this issue, stent expansion behaviour has to be improved by means of an expansion optimized stent design with reduced or in the best case with non-existing dogbone effect. In this work, the influence of stent design modification on its mechanical performance during the implementation has been investigated aiming at the reduction of vessel wall injuries. These investigations include numerical analysis of balloon folding, stent crimping and its free expansion with a folded balloon to quantify the effects of various stents designs on overall and local radial stiffness.. All numerical results are validated by experimental data such as highspeed recordings of stent expansion and collapse, scanning electron microscopy and embedded stents to ensure the realibility of developed approach. The obtained numerical and experimental results prove that already minor stent design modifications such as the increase of strut thickness at the stent ends can prevent the occurence of the undesired dogbone and increase the local and overall radial stiffness of the stent. All results still rely on the free stent expansion. The interaction of modified stents with the vessel wall will be investigated in future studies. 


\section{Effects of transcutaneous spinal cord stimulation on Muscle coordinaton in brain insulted persons}

Thordur Helgason, Health Technology Center, Reykjavik University and Landspitali - University Hospital, Reykjavik, Iceland, thordur@landspitali.is

Vilborg Guðmundsdóttir, Dept. for rehabilitation at Grensas, Landspitali - University Hospital, Reykjavik, Iceland, vilbgu@landspitali.is

Gígja Magnúsdóttir, Dept. for rehabilitation at Grensas, Landspitali - University Hospital, Reykjavik, Iceland, gigjamag@landspitali.is

Rún Friðriksdóttir, Dept. for Science and Engineering, Reykjavik University, Reykjavik, Iceland, run13@,ru.is

Margrét Sól Ragnarsdóttir, Dept. for Science and Engineering, Reykjavik University, Reykjavik, Iceland, margretsr15@ru.is

Belinda Chenery, Dept. for Physiotherapy, University of Iceland, Reykjavik, Iceland, Belinda@styrkurehf.is

Gudbjorg Ludvigsdottir, Dept. for rehabilitation at Grensas, Landspitali - University Hospital, Reykjavik, Iceland, gudbj1@landspitali.is

Transcutaneous spinal cord stimulation (tSCS) has been shown to abbreviate spasticity in lower limbs in people with incomplete spinal cord injury (SCI). This is believed to be due to inhibiting effects on the neural network of the spine at the height of the motor neuron. We therefore hypothesized that the tSCS can influence the spasticity in brain insulted people the same way. In this work we investigate the changes in the electromyogram (EMG) taken in the Wartenberg pendulum test (WPT) and in Achilles tendon stretch test (ATS) before and after tSCS treatment.

Four hemiplegic subjects (man: 67 brain damage, women: 48 stroke, 72 brain tumour, 68 bleeding aneurism) with severe spasm are stimulated in the lumbar area, at the height of th 11 and th12. A 30 minutes posterior root sensory fibres stimulating treatment with $50 \mathrm{~Hz}$ with no EMG responses of the corresponding muscles (sub motor threshold). An assessment of the spasm consisting of EMG measurements and evaluation of passive and active movements was made before, immediately after and four to six hours post tSCS treatment.

Changes in the reflex responses of the muscles are recorded. EMG activity during Wartenberg pendulum test and during Achilles tendon stretch test is changed towards a clear division between the antagonistic muscles. Plantar and dorsiflexion of the foot is done easier. EMG shows less co-contractions and therefore les effort for the movement. Three out of four subject report easier movements. But in the summary the parameters do not indicate a spasticity abbreviation. In contrast to our results with spinal cord injured patients where there was a clear reduction in spasticity, the measurement made in this work on brain insulted subjects do not indicate clearly that tSCS abbreviates spasticity. Three subjects though report less spasticity and improved movement ability. Changes in the EMG from the quadriceps and hamstrings muscles before and after the treatment show a increased coordination between the two antagonistic muscles in WPT. These effects may be the result of increased inhibitory influence through the stimulation of sensory pathways. These effects have to be investigated further. The main difference between the two groups of subjects is that the SCI subjects do have a compromised connection from the brain to the spinal circuitry controlling the muscle whereas the subjects in this study have an intact pathway. 